\title{
Aspectos y enfoques éticos dentro del quehacer conductual
}

\author{
Ethical issues and approaches in the behavioral task
}

\author{
WiLlam Montgomery U. ${ }^{1}$ \\ Facultad de Psicología, UNMSM, Lima Perú \\ RECIBIDO 20/06/2016, ACEPTADO 25/08/2016
}

\begin{abstract}
RESUMEN
En este trabajo se aborda el tema de la ética conductual en sus aspectos conceptual, metodológico y tecnológico. En el plano conceptual se discuten problemas fundamentales y mecanismos explicativos del comportamiento moral. En el plano metodológico uno se refiere a la ética relacionada con la ciencia de la conducta en sí, y otro aborda la ética en las relaciones entre la ciencia del comportamiento y la sociedad. El plano tecnológico implica mantener el contacto entre la investigación experimental y la práctica tecnológica con fundamento científico y buena probabilidad de eficacia, además de seguir protocolos de respeto y protección al cliente.
\end{abstract}

Palabras clave: Ética, psicología, conductismo, teoría, metodología, tecnología.

\begin{abstract}
In this paper the issue of ethical behavior is addressed in its conceptual, methodological and technological aspects. Conceptually fundamental problems and explanatory mechanisms of moral behavior are discussed. At the methodological level one refers to ethics related to behavioral science itself, and another deals with ethics in relations between behavioral science and society. The technological level involves maintaining contact between experimental research and science-based technological practice and good probability of efficacy, while continuing to respect protocols and customer protection.
\end{abstract}

Keywords: Ethics, psychology, behaviorism, theory, methodology, technology.

1 Catedrático de la Facultad de Psicología de la Universidad Nacional Mayor de San Marcos (Lima - Perú), en las asignaturas de Psicología y Técnicas de Intervención Conductuales; Psicología de la Personalidad y Metodología del Trabajo Universitario. E-mail: avidolector@yahoo.es 
Antes de entrar al meollo del tema, que es introducir panorámicamente a los aspectos y enfoques éticos vigentes en el quehacer del psicólogo conductual, se necesita brindar información sobre algunas cuestiones básicas referentes a la psicología como profesión ligada a la ética, y definiciones generales acerca de esta.

Según señala França-Tarragó (2008), la palabra “profesión” implica el ejercicio de una determinada actividad con dedicación y consagración total, así como pertenecer a un grupo de miembros de una sociedad con códigos normados, capacidades y técnicas comunes, y un prestigio social reconocido. Esto obliga a los profesionales a mantenerse fieles a su responsabilidad social y académica. En el caso del psicólogo, él es un profesional comúnmente considerado en los límites de las ciencias sociales y de la salud, por lo cual está íntimamente relacionado con la investigación, el tratamiento y la solución de problemas humanos, lo que le obliga doblemente a ejercer su profesión con responsabilidad ética. Para evaluar sistemáticamente dicho cumplimiento, la ética profesional (o deontología) del grupo (llamado orden profesional) se atiene a criterios objetivos, a la luz de los valores morales predominantes en las relaciones humanas de la comunidad de referencia, y en los conocimientos aportados por la ciencia acerca del comportamiento humano.

En las definiciones aportadas por el Diccionario de la Asociación Americana de Psicología (2009/2010, p. 202) se dice que la ética es: 1) una "rama de la filosofía que investiga tanto el contenido de los juicios morales (es decir, lo que está bien y o que está mal) como su naturaleza (es decir, si dichos juicios deben considerarse objetivos o subjetivos)"; y "2) los principios de la conducta moralmente correcta aceptados por una persona o un grupo o considerados apropiados para un campo específico...". En cuanto a la moral, la misma obra (APA, 2009/2010) consigna que "se relaciona con la distinción entre el bien y el mal o con la conducta que se considera ética o apropiada" (p. 328). Por tanto, como señala Kimmel (2007), "los términos "ética" y "moral" se pueden utilizar indistintamente para referirse a las reglas de conducta apropiada" (p. 6).

En este vasto campo se suelen distinguir dos modalidades: la meta-ética y la ética normativa. Respecto a la meta-ética, a ella le compete aclarar el significado público de los actos como buenos y malos en un ejercicio de "teorización sobre las teorías" acerca de la ética. Por otra parte, la función de la ética normativa es ocuparse de los aspectos prácticos fundamentales de la conducta ética, con el fin de guiarla siguiendo determinados códigos morales. Esta es una "ética general" distinguible también de la "ética aplicada", que surge de las aproximaciones a realidades específicas que cada profesión genera en el marco de su ejercicio práctico, por tanto no está necesariamente de acuerdo con la ética general en ciertos aspectos. Tampoco pretende sustituirla, sino que limita su prescripción a cuestiones problemáticas ligadas a su quehacer, por ejemplo evitando la contaminación "sentimental" de valores humanistas, religiosos, políticos o ideológicos (Chávez, Bustos, Infante y Benavides, 2011).

Una vez consignada la información anterior, es posible ingresar a la consideración del tema central. Así, desde la óptica panorámica utilizada en este ensayo, los 
aspectos éticos en una carrera como la psicología se pueden clasificar en términos conceptuales, metodológicos y tecnológicos, concatenados entre sí y retroalimentados unos por otros, aunque cada uno con sus propios requerimientos aplicativos, y todos subordinados a la responsabilidad ante la ley.

Los niveles de articulación en el paradigma conductual se corresponden con los aspectos reseñados. El nivel superior (conductismo como filosofía) tiene que ver con el plano conceptual, el nivel intermedio (análisis experimental del comportamiento) se refiere al plano metodológico, y el nivel más bajo (ingeniería del comportamiento) empalma con el plano tecnológico. Los tres se retroalimentan entre sí, proveyendo una relación paradigmática entre teoría y práctica, entre ciencia aplicada y ciencia básica, entre filosofía, análisis experimental y tecnología, con el fin de asegurar la utilidad y eficacia de sus procedimientos (Montgomery, 2014).

De acuerdo con el planteamiento anterior, en las secciones siguientes se analizan los aspectos éticos del conductismo separándolos en tres parcelas: el plano conceptual, el plano metodológico y el plano tecnológico. Asimismo, al interior del primero de ellos, se distinguirán enfoques explicativos del comportamiento moral según la variante conductual de que se trate, a saber, mediacional, operante, interconductual y conductual-cognitiva.

\section{ASPECTOS ÉTICOS EN EL PLANO CONCEPTUAL}

Al rubro conceptual le corresponden las definiciones generales de los conceptos que rigen el paradigma teórico-filosófico al cual cada psicólogo se adhiere, y su ética gira en torno a la convicción informada del científico respecto 1) al rigor teórico que le exige escrupulosidad lógica y parsimonia en la interpretación de los hechos que pretende explicar; 2) a su concepción de persona y de sociedad; y 3 ) a la naturaleza de los problemas fundamentales de su disciplina (por ejemplo las relaciones entre libertad y control). Todo eso se verá en los parágrafos sucesivos.

\section{Rigor teórico}

Elinterés porformalizarsistemáticamente lateoríaen términos molares ymoleculares es notorio desde el despliegue de las primeras teorías ligadas al conductismo, siendo el enfoque de Hull-Spence (Gondra, 2007) el más caracterizado en cuanto a esto. Igualmente, Kantor (1967/1978) y Skinner (1953/1971) se esforzaron mucho en esclarecer y organizar el paradigma, lo que también han reimpulsado recientemente Staats (1996/1997) y Ribes (Ribes y López, 1985; Ribes, 2010). Aunque la consolidación total e integrada de estos modelos protosistémicos no ha sido establecida pese a la enorme utilidad conceptual que ellos han tenido como base de investigaciones y aplicaciones, sí se puede afirmar que los intentos son éticamente válidos en cuanto al cumplimiento de la exigencia de la búsqueda pragmática de la verdad, que es la principal función del científico. Sería imposible reseñar en poco espacio un resumen de estas contribuciones, de modo que se dejan apuntadas las referencias. 
Lo que sí es posible es reseñar brevemente las explicaciones metaéticas (y, por tanto, los mecanismos del comportamiento moral) que subyacen a estos enfoques, de entre los cuales se pueden extraer por su claridad los de Eysenck (1964/1976) o de ansiedad condicionada; el de Skinner (1972/1982) o de aprendizaje operante; el de Ribes (1993) o de análisis contingencial; y el de Bandura (1991, 1999) o de modelamiento cognitivo ${ }^{2}$.

Teoría de la ansiedad condicionada. En opinión de H.J. Eysenck, debido a que el niño suele tomar lo que quiere o necesita prescindiendo de los derechos de los demás, es entrenado para tener en cuenta los derechos y deseos de los otros. Este proceso está presidido por los padres, los maestros, los compañeros y otras personas significativamente relacionadas con el infante, que operan como "supervisores de la moralidad". De esta manera, cualquier cosa considerada por ellos como equivocada o antisocial que el niño cometa será castigada de alguna forma, implantando diversos grados de ansiedad condicionada cuya supresión sería la que evita la emisión de respuestas antisociales. El proceso sería similar al del condicionamiento clásico, donde la actividad antisocial es el estímulo condicionado (campana en el experimento pavloviano) y el castigo es el estímulo incondicionado (similar a la comida en el mismo experimento). Así, mediante la asociación de la ocurrencia de los actos antisociales y el castigo durante un determinado número de veces, se condiciona al niño a esperar el castigo y a experimentar el miedo y la ansiedad asociados a él en relación con la actividad antisocial. A esto se le puede llamar una "conciencia moral" que "bloquea" la emisión de respuestas instrumentales antisociales en un proceso de evitación pasiva. La sola posibilidad de emisión de una respuesta delictiva que anteriormente haya estado apareada con el castigo, genera gran volumen ansiógeno (estímulo aversivo) que se suprime al evitarse la citada respuesta, lo cual, a su vez, refuerza negativamente el no cometerla y de esa forma aumenta la probabilidad de no emisión de conductas delictivas. Los casos en los cuales este condicionamiento no se consolida suelen producirse en función a condiciones especiales de crianza o a temperamentos de orden inestable no susceptibles al castigo.

Teoría del aprendizaje operante. Para Skinner, el término ética se refiere a ciertos estándares de conducta que se desarrollan en una cultura y promueven su supervivencia. En este sentido, la ética es una clase de conducta social posiblemente funcional desde que resultó naturalmente reforzante el que la conducta de un ser humano tenga efectos sobre la de los demás. En este devenir, se comenzó a reforzar la conformidad a ciertos patrones y a castigar a quienes se desviaban de lo establecido. Al evolucionar tales contingencias sociales, se consolidaron entidades que asumieron el poder de controlar mediante el castigo y la amenaza de castigo y aparecieron las normas y leyes. Estas reglas describen relaciones de contingencias concebidas para el autocontrol ético de grupo, a fin de evitar beneficios excesivos de quienes mantienen las relaciones conducta-consecuencias (las autoridades)

2 Para el esclarecimiento de dichas propuestas también se han consultado textos que sintetizan parte de su formulación (Chiesa, 1993; Rodríguez, 1995; Clavijo, 1998; Ballesteros, 2000; Pallejá, Garrido y Herrero, 2004;y Martin y Pear, 1996/2008). 
por encima de quienes son afectados por ellas. Por ello, las constituciones, legislaciones y otros documentos políticos pueden considerarse especificaciones formales de contingencias diseñadas para controlar el comportamiento de aquellos que controlan el comportamiento de los demás (conducta guiada por reglas). De hecho, cuando un individuo se conduce de una forma que genera estímulos punitivos para otros miembros de una cultura, las pautas éticas se convierten en fuente importante de control conductual. En este contexto, los valores surgen determinados por procesos de selección sobre la base de contingencias de tipo social especificadas a través de reglas, puesto que la comunidad verbal es la que selecciona qué conductas son "correctas" para reforzarlas positivamente, al mismo tiempo que determina contingencias punitivas para las conductas "no-correctas".

Teoría del análisis contingencial. El análisis contingencial es, dentro de la teoría interconductista, un sistema analítico-evaluativo de la valoración del comportamiento individual. Desde esta óptica, la moral viene a ser una dimensión de la propia conducta en correspondencia entre las prácticas valorativas de un individuo y las de su comunidad de referencia. Tales prácticas son reguladas por condiciones no presentes en las situaciones en que se despliegan, y pueden presentarse de variadas formas en relación con el "deber ser" del comportamiento. La estructura del análisis contingencial consta de dos ejes: a) el sistema macrocontingencial como conjunto de prácticas supraordinado a las situaciones, que define sus criterios valorativos; y b) el sistema microcontingencial como red de relaciones interindividuales que median la interacción socialmente valorada en una contingencia. El cruce de ambas permite el análisis de correspondencias entre dos tipos de microcontingencias: las de naturaleza situacional o valoradas como problemáticas, y las de naturaleza ejemplar bajo las que se explicitan los juicios de valor moral como "deber ser" de las relaciones. Las situaciones de correspondencia en términos de regulación moral por parte de la comunidad pueden ser once: a) prescripción, si se modela o instruye sobre una clase de relación; b) condicionamiento, si se instruye sobre los requerimientos a cumplir previos a una relación;c) indicación, si se señala una opción sobre otra; d) comparación, si se contrastan dos formas de relación; e) facilitación, si se auspician o disponen las condiciones para que se dé una relación; f) expectativa, si se instruye sobre las demandas sociales que una relación debe satisfacer; g) prohibición, si se señala la imposibilidad de una conducta; h) sanción, si se operan consecuencias concretas para la relación ; i) justificación, si se instruye o modela sobre las consecuencias que sigue una relación; j) advertencia, si se señalan las consecuencias que pueden ocurrir dada la relación; y k) omisión, si se desatiende o se omite alguna explicitación sobre la conducta.

La moralidad, según Ribes, consiste en una regulación de modos de comportamiento que corresponden a otros en términos de propiedades convencionales (ejemplares), como prácticas individuales de acuerdo con la posición relativa que ocupan en el grupo social. 
Teoría del modelamiento cognitivo. En las explicaciones eysenckiana y skinneriana ya se ve que la imitación social es un medio efectivo para establecer conductas. Bandura la resitúa en primer lugar y la corona con la mediación explícita de procesos cognitivos. Para él, las sanciones y justificaciones que proporcionan los modelos paternos y simbólicos son fuentes de juicios morales a través del aprendizaje social (modelado y refuerzo), pero la determinación no es automática ni directa. Los repertorios que dirigen la conducta son aquellos guiados por juicios morales, que operan como una autocensura de carácter anticipatorio que evita que la gente transgreda normas. Por lo tanto, el desarrollo de los juicios morales superiores se alcanza cuando las personas juzgan los acontecimientos en función de principios previamente aprendidos. En este sentido, la teoría sociocognitiva de Bandura afirma que las personas poseen una función de agencia planificadora y autorreguladora (autoinstruccional), generada por el interjuego entre el sistema y la influencia del ambiente (modos de refuerzo y de presentación del modelo, efectos del castigo y prácticas de manejo familiar), que les permite ejercer el control sobre sus pensamientos, sentimientos, motivación y acciones.

En 1999, Bandura propuso también su "teoría de la desconexión moral”, que intenta explicar el proceso mediante el cual las sanciones personales pueden desvincularse de las conductas inmorales e inhumanas. Señala que las influencias de la reacción personal (como "autosanción" moral) no actúan al menos que sean activadas, y cada individuo aprende a discriminar en qué circunstancias puede desactivarlas o no con el fin de evitarse conflictos entre el "deber" y el "hacer". Los mecanismos de la desconexión moral son divididos en cuatro categorías: a) los referidos a la conducta misma procuran rebautizarla para que no se entienda como inhumana, justificándola moralmente; comparándola con alternativas peores; o utilizando eufemismos; b) los referidos al desplazamiento de la responsabilidad, echándole la culpa a otros o difuminando la responsabilidad entre muchos; c) los referidos al distanciamiento entre la acción y sus efectos, ignorando las consecuencias del acto inhumano; y d) los referidos a la víctima de la acción inmoral tienen que ver con la deshumanización de aquella o el echarle la culpa de lo que pasa.

Una conclusión "integrativa”. ¿Hasta qué punto los modelos explicativos reseñados discrepan entre sí, más allá de la jerga técnica que utilizan? La figura 1 muestra un ejemplo tentativo de conducta moral en la que alguien emite una respuesta que podría considerarse compatible con todas las teorías presentadas, pues los principios que cada cual formula están presentes de una u otra forma.

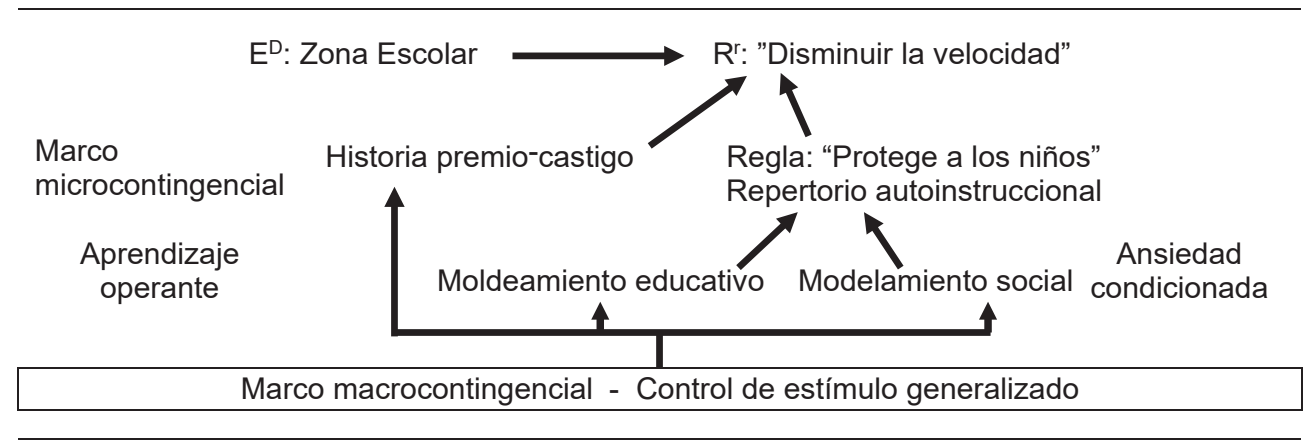


Figura 1. Conducta moral de un chofer manejando en una zona escolar.

\begin{tabular}{|c|c|}
\hline & ED: Zona Escolar R": "Disminuir la velocidad" \\
\hline $\begin{array}{l}\text { Marco } \\
\text { microcontingencial }\end{array}$ & $\begin{array}{l}\text { Historia premio-castigo Regla: "Protege a los niños" } \\
\text { Repertorio autoinstruccional }\end{array}$ \\
\hline Aprendizaje operante & Moldeamiento educativo modelamiento social Ansiedad condicionada \\
\hline
\end{tabular}

Así, quizá puede entenderse que el marco más general o de base lo constituirían las macrocontingencias, proporcionando un control de estímulo generalizado en la terminología clásica del análisis de la conducta. A su vera se desarrollan los procesos por conducta respondiente y operante, entre los cuales están el moldeamiento educativo, el modelamiento social y las prácticas sociales premio-castigo. Luego, en el momento de la confrontación entre este aprendizaje (ejemplar) y la situación microcontingencial específica (estímulo discriminativo $\left[\mathrm{E}^{\mathrm{D}}\right]$ de guía por reglas establecidas en la comunidad de referencia), el sujeto toma una decisión ética autoinstruccional (respuesta históricamente reforzada $\left[\mathrm{R}^{\mathrm{r}}\right]$ ) confrontando ambos.

\section{Concepción de persona y de sociedad}

Desde el punto de vista de Skinner (1972/1982), que es el único autor conductual relevante que se ha esforzado por ofrecer amplias nociones explícitas al respecto, la concepción tradicional de "hombre autónomo" no deja alternativas para lograr cambios provechosos para la humanidad, porque impide que la conducta humana sea objeto de estudio de la ciencia por derecho propio. A pesar de que es teóricamente posible partir del análisis del origen de los actos humanos desde la persona, considera que dicha opción ya ha probado ser ineficaz, de modo que se necesita creer pragmáticamente en el determinismo del comportamiento, pues, por otra parte, la realidad del control ambiental no puede ser negada (Skinner, 1953/1971). En este sentido, hay tres grandes determinantes del comportamiento que se deben tener en cuenta: el primer determinante se encuentra en las contingencias filogenéticas (responsables de las características del individuo como miembro de una especie); el segundo está compuesto por las contingencias que seleccionan y fortalecen aquellos comportamientos que producen efectos reforzantes, haciendo más probable la ocurrencia futura de esas clases de conducta; y el tercero se refiere a las contingencias de refuerzo social que ayudan a explicar la supervivencia de las prácticas culturales.

Por estas razones, la selección por consecuencias filogenéticas y ontogenéticas es el tipo de explicación causal para Skinner. En este marco, la persona es un organismo biológico en interacción con un ambiente social: un sistema de comportamiento muy complejo que implica, a su vez, un conjunto de repertorios de conducta culturalmente construidos. Nos convertimos en personas desde el momento en que 
empezamos a interactuar con otras, formando parte de una comunidad verbal que comparte prácticas culturales y lenguaje. Lo que se llama "individualidad" surge de las contingencias del contexto donde interactuamos a lo largo de la vida, en confluencias únicas e irrepetibles para cada persona. Estas características permiten inferir una "antropología" no reduccionista y pro-humanista, en el sentido de imbricación hombre-sociedad mutuamente incluyentes.

\section{Libertad y Control de la Conducta}

Al no poder ser negada la realidad del control, según lo dicho en el parágrafo anterior, el objetivo ético es lograr que todos seamos analistas de nuestro propio comportamiento, para garantizar que podamos discriminar repertorios conductuales que sirvan de control de otros repertorios conductuales, y contingencias que los influencien. Eso significa capacitar a la persona para que aprenda habilidades de autocontrol verbal y no verbal, promoviendo la auto-observación y automedición respecto a su funcionamiento cognitivo, afectivo, motor, fisiológico y somático (así como a la fuerza de su repertorio); e incentivar el análisis funcional de su propia conducta en relación con su medio ambiente particular. En este sentido, se puede afirmar que lo que el individuo experimenta como "libertad" en ciertas situaciones no es más que la falta de restricción sobre su comportamiento, lo que se logra enseñándole a manejar las contingencias convenientemente para cambiar su entorno. En la mayoría de casos, esto significa enseñarle a usar su conducta verbal.

Lo cierto es que, a nivel humano, el carácter de las contingencias es mediado por los procesos lingüísticos y simbólicos (formando lo que algunos llaman "marcos relacionales"). El lenguaje, al posibilitar mensajes acerca de objetos y sucesos que no están presentes, e incluso que no existen, crea campos de interacción que simulan un mundo real, como cuando la gente habla de pensamientos, deseos, sentimientos y otras eventualidades, atribuyéndoles una existencia independiente de las condiciones del entorno que provocan sus efectos. Skinner (1957/1981) especifica que en esta clase de construcción se utiliza principalmente la conducta verbal autoclítica: aquella que constituye también parte del ambiente con el que interactúa la persona, emitiendo tactos sobre otros $\operatorname{tactos}^{3}$ para referirse a respuestas potenciales, discriminativas, de efectos en presente y futuro, a variables que controlan la conducta y al grado de probabilidad que tienen. En la figura 2 se presentan ejemplos de esas respuestas.

3 Operantes verbales que están bajo el control de estímulos no verbales. Pueden ser abstractas (como cuando se hipotetizan ocurrencias o estados potenciales de conductas ajenas en base a indicios), o concretas (como cuando se rotulan objetos). 
Figura 2. Ejemplos de conductas verbales autoclíticas

\begin{tabular}{ll}
\hline \multicolumn{1}{c}{ Conducta autoclítica } & \multicolumn{1}{c}{ Ejemplo } \\
\hline Potencial & $\rightarrow$ "Si quisiera, yo podría llegar a ser un santo" \\
Discriminativa & $\rightarrow$ "Somos seis hermanos en total" \\
Efectos en presente/futuro $\rightarrow$ "Dentro de un mes viajaré al interior" \\
Variables controlantes & $\rightarrow$ "Prefiero dinero en efectivo antes que un vale de cambio por mercadería" \\
Probabilidad de ocurrencia & $\rightarrow$ "Quizá hoy podré pedirle un aumento de sueldo a mi jefe" \\
\hline
\end{tabular}

Gracias a las posibilidades abiertas por la conducta verbal, entonces, el individuo humano experimenta la espontaneidad, la libertad, la autodirección y la actividad constituidas por la forma con que los individuos amplían su aprendizaje pasado mediante el razonamiento, planeación, metas con un propósito determinado, etcétera. Como dice Staats (1975/1979), "esta conciencia de la propia autodeterminación puede considerarse como un epifenómeno. Surge de las acciones de los repertorios de personalidad aprendidos del individuo y de los efectos que estas acciones tienen" (p. 430). Así se resuelve la paradoja entre libertad y control.

\section{ASPECTOS ÉTICOS EN EL PLANO METODOLÓGICO}

Al rubro metodológico le corresponde prestar atención al grado suficiente de exhaustividad al analizar empíricamente los objetos y datos de estudio siguiendo los pasos del método científico, asegurar la validez y confiabilidad de materiales e instrumentos utilizados para su medición, optar por diseños que sean adecuados al tipo de fenómenos investigados y formular los principios que dan cuenta de las regularidades encontradas en la investigación empírica.

Este tema se puede subdividir en dos aspectos: uno referente a la ética relacionada con la ciencia de la conducta en sí, que comprende: a) la exhaustividad del investigador conductual al analizar empíricamente su objeto de estudio; b) el aseguramiento de la validez y confiabilidad de los materiales e instrumentos de medición y evaluación; y c) el rigor investigativo a través de los procedimientos experimentales. Otro aborda la ética en las relaciones entre la ciencia del comportamiento y la sociedad, que incluye: d) la responsabilidad moral de los científicos de la conducta acerca del uso que se le da a sus investigaciones; y e) la manera de enfocar investigaciones que involucran posibles violaciones a principios éticos. Estos puntos se desarrollan a continuación.

\section{La exhaustividad al analizar empíricamente el objeto de estudio}

Las diferentes variedades conductuales se preocupan por hacer de la ciencia del comportamiento un método analítico extremadamente detallado y preciso, 
entendiendo que este es indispensable para llevar a cabo las tareas del psicólogo científico. Esto se cumple en el "análisis experimental del comportamiento", donde se formulan las categorías, las unidades analíticas, los parámetros, los paradigmas de investigación, y las leyes y principios implicados en el manejo de los datos. Deben destacarse aquí las ecuaciones que cubren todo el espectro organísmico y situacional implícito o explícito en una contingencia. La más conocida de dichas ecuaciones es la comprendida en la fórmula: $\mathrm{K}=f[\mathrm{E}-\mathrm{O}-\mathrm{R}-\mathrm{C}]$, que significa que un segmento contingencial $(\mathrm{K})$ es función $(f)$ de las interrelaciones establecidas entre los factores estimulares (E), organísmico-disposicionales (O), de respuesta o clases de respuesta respondientes y operantes $(\mathrm{R})$, y las consecuencias que fortalecen a estas últimas $(\mathrm{C})$.

\section{Aseguramiento de validez y confiabilidad de materiales $e$ instrumentos}

El proceso de evaluación, como lo señalan Fernández-Ballesteros y Calero (2004), se sujeta a los estándares éticos suministrados por las asociaciones científicas internacionales, aplicando normas y recomendaciones para la revisión de los índices de validez y confiabilidad de pruebas psicológicas, que son muy frecuentes hoy en la ejecutoria psicométrica (American Psychological, Asociation, 2010). Esto con mayor razón cuanto en los últimos tiempos se ha producido una "reconciliación" de la evaluación conductual funcional con los métodos psicométricos de corte estadístico, proporcionando muchas herramientas evaluativas novedosas.

\section{Rigor investigativo a través de los procedimientos experimentales}

Implica seguir los pasos del método científico formulando el problema con precisión. Proponer conjeturas definidas y fundadas, sometiéndolas a contrastación dura a través de la opción por diseños experimentales, privilegiando procedimientos estratégicamente inductivos y cuantitativos que aseguren la descripción objetiva y confiable de cada respuesta bajo estudio. Estos procedimientos incluyen el control experimental sobre las variables independientes que pueden determinar el comportamiento (variable dependiente), minimizando el papel de las variables extrañas. La aplicación de este manejo investigativo en aras de la búsqueda de la verdad ha alcanzado a los formatos de publicación de la Asociación Psicológica Americana; haciéndolos cada vez más objetivos y rigurosos en cuanto a su estilo y redacción. Referente al cuidado ético que hay que tener en la investigación conductual con sujetos humanos y animales, Kimmel (2007) presenta un detallado análisis de las diferentes condiciones de información (consentimiento informado), transparencia, respeto y justicia que son indispensables en semejante labor, recalcando que para la actual generación de estudiantes de la ciencia del comportamiento las cuestiones éticas son ahora parte de un marco más amplio de cuestiones científicas, en beneficio de su formación crítica como futuros investigadores. 


\section{Responsabilidad moral acerca del uso que se le da a las investigaciones}

La investigación de la conducta, a través del control de contingencias de reforzamiento, está relacionada con el cambio de la probabilidad de que los organismos se comporten de ciertas maneras. En este sentido, es una manera ética especial de ayudar a la gente, puesto que se plantea cuestiones como las siguientes: "¿Podemos diseñar un medio ambiente en el cual la gente se trate bien, mantenga la población dentro de ciertos límites, aprenda a trabajar productivamente, preserve y estimule el carácter reforzante del mundo, explore y analice ese mundo, limite el uso de recursos y mantenga a salvo el medio ambiente para generaciones futuras, y haga todo esto porque los resultados son reforzantes en forma positiva?" (Skinner, 1978/1982, p. 10). Sin embargo, la objeción al desarrollo de una ciencia que aborde esos problemas es que apela al control del ser humano, y ¿quién controlaría a los controladores?; pero la respuesta de Skinner es que en un diseño ambiental mejorado los propios controladores estarían sujetos a las prácticas culturales inducidas.

\section{Manera de enfocar investigaciones que involucran posibles violaciones a principios éticos}

El quehacer investigativo con sujetos humanos y animales que surge del análisis experimental del comportamiento actualmente se sujeta a marcos legales de la comunidad y normativos de la disciplina, que limitan su desempeño de acuerdo con lo que convencionalmente se tolera hoy como prácticas aceptables de experimentación. La APA (2010) tiene su propio reglamento ético, y hay también instituciones locales (por ejemplo, los colegios de psicólogos de cada región) que regulan estas prácticas, de modo que no sean violatorias de derechos humanos o animales fundamentales. Por ejemplo, bajo las reglas actuales, una investigación cuestionable como la hecha por Watson con el niño Albert no sería posible (ver Digdona, Powell, \& Smithson, 2014; sobre el punto de cuánto hay de verdad en esa historia).

\section{ASPECTOS ÉTICOS EN EL PLANO TECNOLÓGICO}

Finalmente, al rubro tecnológico le corresponden, por un lado, mantener el contacto entre la investigación experimental y la práctica clínica con el fin de elaborar técnicas con fundamento científico y buena probabilidad de eficacia, y por otro, seguir protocolos de respeto y protección al cliente promoviendo interacciones terapéuticas de mutuo control en todas las ramas de la atención psicológica.

Los rubros a tratar en esta sección involucran, entre las más importantes: a) mantener un contacto estrecho entre investigación experimental y aplicaciones; $b$ ) seguir protocolos de respeto y protección al cliente; c) llevar a cabo interacciones terapéuticas de mutuo control; y d) privilegiar intervenciones de terapia y modificación de conducta con apoyo empírico. A continuación, se desarrollan esos puntos. 


\section{Contacto estrecho entre investigación experimental y aplicaciones}

Es éticamente deseable que la ciencia básica se entronque de manera coherente con la ciencia aplicada, dejando claros los principios que fundamentan las intervenciones en cualquier área; por ello, el análisis conductual se esfuerza constantemente en proveer dicha coherencia. Posiblemente, la mayoría de los psicólogos estarían de acuerdo en que una disciplina científica necesita mostrar una articulación paradigmática coherente entre teoría y práctica, entre ciencia aplicada y ciencia básica, entre análisis experimental y tecnología, con el fin de asegurar la utilidad y eficacia de sus procedimientos.

Los paradigmas de investigación centrales desplegados desde el análisis experimental son los del condicionamiento clásico y del condicionamiento operante, así como de sus diversas combinaciones y formas de presentación (por ejemplo los programas de reforzamiento). Estos paradigmas son equivalentes a los "ejemplares" de Kuhn, puesto que desde sus modos básicos de ocurrencia empírica se desprenden leyes, teorías, aplicaciones e instrumentación juntas. Gracias a la investigación en estos rubros se han obtenido una gran cantidad de regularidades de la conducta, que pueden considerarse como leyes que desembocan en la formulación de principios aplicativos a nivel operante y respondiente (como, por ejemplo, el reforzamiento, la extinción, el castigo y el contracondicionamiento). La formulación de algunas de estas leyes se presenta en la tabla 1 a manera de ejemplo, con el fin de probar el entronque básico-aplicado que existe entre los niveles del análisis experimental y la tecnología del comportamiento.

Tabla 1. Ejemplos de regularidades de la conducta que son fundamento de prácticas aplicativas.

\begin{tabular}{ll}
\hline \multicolumn{1}{c}{ Principio } & Ejemplo de aplicación \\
\hline $\begin{array}{l}\text { Efecto: "De entre varias respuestas emitidas en la misma situación, } \\
\text { aquella seguida o acompañada de ciertas consecuencias para el } \\
\text { organismo tendrá una mayor probabilidad de ocurrencia en circuns- } \\
\text { tancias similares". }\end{array}$ & $\begin{array}{l}\text { Técnicas de modificación } \\
\text { de conducta }\end{array}$ \\
$\begin{array}{l}\text { Saciedad: "Si un reforzador se presenta continuamente durante al- } \\
\text { gún tiempo, la tasa de respuestas asociada a él tenderá a reducirse". }\end{array}$ & Sobrecorrección \\
$\begin{array}{l}\text { Privación: "Si se retira un reforzador durante algún tiempo, la tasa } \\
\text { de respuestas asociada a él se eleva primero y luego se reduce". }\end{array}$ & Extinción operante \\
$\begin{array}{l}\text { Sobreposición: "Si se coloca gradualmente un estímulo nuevo sobre } \\
\text { otro con función discriminativa, el control se transfiere al primero". }\end{array}$ & Moldeamiento \\
$\begin{array}{l}\text { Inhibición: "La fuerza de una respuesta se puede reducir mediante } \\
\text { la presentación de un segundo estímulo que, por sí mismo, no tiene } \\
\text { ninguna relación con el efecto en cuestión". }\end{array}$ & Extinción respondiente \\
$\begin{array}{l}\text { Preponderancia: "Cuando dos o más respuestas se sobreponen, una } \\
\text { de ellas tenderá a ocurrir excluyendo a la otra". }\end{array}$ & Reacción de competencia \\
$\begin{array}{l}\text { Premack: "La respuesta cuya ocurrencia sea más probable tiende a } \\
\text { reforzar otra de menor probabilidad". }\end{array}$ & Juegos educativos \\
\hline
\end{tabular}




\section{Respeto y protección al cliente}

Los buenos terapeutas de conducta deben estar interesados en sus clientes como personas. Aquellos que muestran un comportamiento que parece ser frío y mecánico no se encuentran con más frecuencia en el campo de la modificación de conducta que en cualquier otra forma de asistencia profesional. Las cuestiones relacionadas con la aplicación ética de la tecnología conductual son, de acuerdo con Martin y Pears (1996/2008), fijadas por la Asociación para el Avance de la Terapia de Conducta (AACT), la Asociación de Psicología Americana (APA) y la Asociación para el Análisis de Conducta (AAC). Esta última ha formulado una serie de pautas para supervisar las intervenciones:

- ¿Se han meditado adecuadamente los objetivos del tratamiento?

- ¿Se ha meditado adecuadamente la selección de los métodos de tratamiento?

- ¿Participa el cliente voluntariamente?

- ¿Se ha evaluado la eficacia del tratamiento?

- ¿Se ha protegido la confidencialidad de la relación terapéutica?

- ¿De ser necesario, remite el terapeuta a sus clientes a otros terapeutas?

- ¿Está cualificado el terapeuta para aplicar el tratamiento?

\section{Interacciones terapéuticas de mutuo control}

Para garantizar el trato ético entre el modificador y el cliente deben organizarse contingencias con propiedades de contracontrol; es decir, la influencia que la persona controlada tiene sobre la que controla, a través del acceso a los reforzadores adecuados. Un cliente puede dejar de ver a un terapeuta como forma de contracontrol, asegurándose así de que el terapeuta se adherirá a las pautas de tratamiento previamente establecidas. Otra medida es que el modificador de conducta debe indicar claramente al cliente cuáles son sus acreditaciones, y su cumplimiento de requisitos profesionales exigidos por las instituciones oficiales. Al fijarse objetivos de tratamiento, estos también deben ser coherentes con los derechos básicos (dignidad, intimidad y cuidado humanitario) del cliente. Los objetivos deben ser coherentes también con el bien de la sociedad. A este respecto, el cliente debe ser un participante activo a la hora de seleccionar los objetivos e identificar las conductas meta. Finalmente, hay que estipular que no todo programa tiene que formularse con el consentimiento del cliente para participar en él, basado en el conocimiento de los procedimientos que se van a utilizar, y de sus probables efectos. Ello comprende discutir con el usuario del servicio los datos que evalúan su progreso a lo largo de todo el programa. 


\section{Intervenciones con apoyo empírico}

Como es obvio, los modificadores de conducta deben recibir entrenamiento práctico para asegurar su competencia en la evaluación, diseño y programación del tratamiento y su evaluación, pero también deben tener acceso a la información que les permita conocer cuáles tratamientos son los mejores, y decidir éticamente sobre la utilización de los métodos más eficaces, empíricamente validados, que provoquen las menores molestias y efectos secundarios negativos. Fue Eysenck uno de los pioneros en defender la realización de estudios rigurosos sobre la eficacia de los tratamientos psicológicos y que la práctica clínica debería sustentarse en los resultados de tales investigaciones. Eso, con el tiempo, se ha denominado "práctica clínica basada en la evidencia" a semejanza de lo hecho en la carrera médica, sobre la base de la necesidad de estandarizar tratamientos por medio de protocolos para ser utilizados globalmente con la misma eficacia y eficiencia. Esta labor, entonces, busca difundir la aplicación de los tratamientos psicológicos que han sido sometidos a prueba científica con buenos resultados; y después de muchos estudios se encuentra que la mayor parte de los tratamientos eficaces en psicología son los derivados del modelo cognitivo-conductual (Mustaca, 2004).

\section{CONCLUSIONES}

1. A manera de estructuración general tentativa de su papel dentro del quehacer conductual, la ética se puede analizar en tres grandes aspectos: conceptual, metodológico y tecnológico.

2. El nivel conceptual comprende problemas fundamentales (concepción de persona y de sociedad, relaciones entre libertad y control), y mecanismos explicativos del comportamiento moral. Dentro de estos últimos están los enfoques de ansiedad condicionada; el de aprendizaje operante; el de modelamiento social; y el de análisis contingencial.

3. El nivel metodológico comprende pautas como: a) la exhaustividad del investigador conductual al analizar empíricamente su objeto de estudio; b) el aseguramiento de la validez y confiabilidad de los materiales e instrumentos de medición y evaluación; c) el rigor investigativo a través de los procedimientos experimentales; d) la responsabilidad moral de los científicos de la conducta acerca del uso que se le da a sus investigaciones; y e) la manera de enfocar investigaciones que involucran posibles violaciones a principios éticos.

4. El nivel tecnológico comprende pautas como: a) mantener un contacto estrecho entre investigación experimental y aplicaciones; $b$ ) seguir protocolos de respeto y protección al cliente; c) llevar a cabo interacciones terapéuticas de mutuo control; y d) privilegiar intervenciones de terapia y modificación de conducta con apoyo empírico. 


\section{REFERENCIAS BIBLIOGRÁFICAS}

American Psychological, Asociation (2010). Ethical principles of psychologists and code of conduct. Recuperado de: https://www.apa.org/ethics/code/ principles.pdf

Asociación de Psicología Americana (2009/2010). Diccionario Conciso de Psicología. México: El Manual Moderno.

Ballesteros, B.P. (2000). Ética y sociedad en el pensamiento de B. F. Skinner. Suma Cultural, 1(2), 123-149.

Bandura, A. (1991). Social cognitive theory of moral thought and action. In W.M. Kurtines y J.L. Gewirtz(eds.). Handbook of moral behavior and development (Vol. 1, pp. 45-101). Hillsdale, N.J.: Lawrence.

Bandura, A. (1999). Moral disengagement in theperpetration of inhumanities. Personality and Social Psychology Review, 3, 193-209.

Bunge, M. (1996). Ética, Ciencia y Técnica. Buenos Aires: Editorial Sudamericana.

Clavijo, A. (1998). Etica y comportamiento: Una perspectiva conductista. Suma Psicológica, 5(1), 65-85.

Chávez, G., Bustos, A.G., Infante, J.M. y Benavides, B. (2011). Ética, sociedad y profesión. México: Grupo Editorial Patria.

Chiesa, M. (2003). Sobre la meta-ética, la ética normativa y el conductismo. Revista Latinoamericana de Psicología, 35(3), 289-297.

Digdona, N., Powell, R.A. \& Smithson, Ch. (2014). Watson's alleged Little Albert scandal: Historical breakthrough or new Watson myth? Revista de Historia de la Psicología, 35(1), 47-60.

Eysenck, H.J. (1964/1976). Delincuencia y personalidad. Madrid: Marova.

Fernández-Ballesteros, R. y Calero, M.D. (2004). Garantías científicas y éticas de la evaluación psicológica. En R. Fernández-Ballesteros (ed.). Evaluación psicológica: Conceptos, métodos y estudio de casos (pp. 121-158). Madrid: Pirámide.

França-Tarragó, O. (2008). Ética para psicólogos. Introducción a la psicoética (6ta. ed.). Bilbao: Descleé de Brouwer.

Gondra, J. M. (2007). Mecanismos asociativos del pensamiento. La "obra magna" de Clark L. Hull. Bilbao: Desclée de Brouwer.

Kantor, J.R. (1967/1978). Psicología interconductual. Un ejemplo de construcción científica sistemática. México: Trillas.

Kimmel, A.J. (2007). Ethical issues in behavioral research: Basic and applied perspectives. Cambridge, M.A.: Blackwell Publishing. 
Martin, G. y Pear, J. (1996/2008). Modificación de conducta. Madrid: PrenticeHall.

Montgomery, W. (2014). Teoría, evaluación y cambio de la conducta. Lima: Asociación de Psicología Teórica, Tecnológica y Aplicada Internacional.

Mustaca, A.E. (2004). Tratamientos psicológicos eficaces y ciencia básica. Revista Latinoamericana de Psicología, 36, 1, 11-20.

Pallejá, J.M., Garrido, E. y Herrero, M.C. (2004).Teoría social cognitiva de la conducta moral y de la delictiva. EnF. Pérez Álvarez (coord.): Serta: In memoriam Alexandri Baratta (pp. 379-414).Salamanca: Ediciones Universidad de Salamanca.

Ribes, E. (1993). El análisis contingencial y la identificación y definición funcional de los problemas psicológicos. Revista Mexicana de Psicología, 10(1), 8589.

Ribes, E. (2010). Teoría de la conducta 2. Avances y extensiones. México: Trillas.

Ribes, E. y López, F. (1985). Teoría de la conducta. Un análisis de campo y paramétrico. México: Trillas.

Rodríguez, M. (1995). La dimensión moral de la conducta desde una óptica interconductual. Acta Comportamentalia. 3(1), 55-69.

Skinner, B.F. (1953/1971). Ciencia y conducta humana. Barcelona: Fontanella.

Skinner, B.F. (1957/1981). Conducta verbal. Barcelona: Fontanella.

Skinner, B.F. (1972/1982). Más allá de la libertad y la dignidad. Barcelona: Fontanella.

Skinner, B.F. (1978/1982). Conducta humana y democracia. En B.F. Skinner. Reflexiones sobre conductismo y sociedad (pp.7-17). México: Trillas.

Staats, A.W. (1975/1979). Conductismo social. México: El Manual Moderno.

Staats, A.W. (1996/1997). Conducta y personalidad. Bilbao: Desclée de Brouwer. 